\title{
El "peine fino" sería más efectivo que los pediculicidas
}

\author{
"Thin comb" seams to be more effective than pediculicide drugs
}

N Hill N y col. BMJ 2005; 331:384-387.

\section{Objetivo}

Comparar la efectividad del uso sistemático del peine fino (PF) con el tratamiento simple con pediculicidas para eliminar los piojos.

\section{Diseño}

Ensayo clínico aleatorizado simple ciego.

\section{Lugar}

Cinco condados de Inglaterra y Escocia.

\section{Reclutamiento}

Pósteres en farmacias y escuelas y reuniones de padres.

\section{Criterio de inclusión}

Mayores de dos años con diagnóstico confirmado por el médico de familia y/o la enfermera de la comunidad y que en las últimas semanas no hubieran realizado tratamiento alguno para la pediculosis ("caso índice*"). Se examinó al resto de la familia indicándoles el mismo tratamiento si estaban infestados. Sólo el caso índice fue analizado.

\section{Intervención}

El dispositivo cazabichos incluyó un PF y las instrucciones para su uso: usar el peine fino cada tres días sobre el cabello mojado con acondicionador, hasta completar cuatro veces. El dispositivo pediculicidas estaba compuesto por una solución de permetrina acuosa al $1 \%$ o de malatión $0,5 \%$. No se entregaron instrucciones adicionales a las detalladas en el envase de cada producto.

\section{Medición de resultados principales}

Presencia de piojos luego de dos a cuatro días de finalizado el tratamiento. Con el objetivo de que tuvieran la misma probabilidad de reinsfestación fueron evaluados al quinto día los que utilizaron pediculicidas y al décimo quinto los asignados al PF. El estudio contó con un poder superior al $80 \%$ para detectar un $30 \%$ de diferencia entre ambos grupos.

\section{Resultados}

Los registros de venta de las 92 farmacias del área de estudio informaron sólo un $8 \%$ de superposición de ambas ramas. Los principales resultados y el análisis de sensibilidad ${ }^{\star}$ se muestran en la tabla 1.

Tabla 1: presencia de piojos a los tres días de finalizado el tratamiento y análisis de sensibilidad.

\begin{tabular}{|c|c|c|c|c|c|c|}
\hline & \multicolumn{3}{|c|}{ Número de pacientes } & \multirow[b]{2}{*}{$\begin{array}{c}\% \text { de } \\
\text { curación } \\
\text { y número }\end{array}$} & \multicolumn{2}{|c|}{ RR (IC95\%) } \\
\hline & Aleatorizados & Perdidos & $\begin{array}{c}\text { Que } \\
\text { completaron } \\
\text { el tratamiento }\end{array}$ & & $\begin{array}{l}\text { Resultados } \\
\text { observados }\end{array}$ & $\begin{array}{l}\text { Peor escenario } \\
\text { para el peine } \\
\text { fino }^{3}\end{array}$ \\
\hline Peine fino & 62 & 6 & 56 & $57 \%$ (32) & $4,4(2,3-8,5)$ & $3,7(2,0-6,8)$ \\
\hline Pediculicida & 71 & 1 & 70 & \begin{tabular}{|l|}
$13 \%(9)$ \\
\end{tabular} & 1 & 1 \\
\hline
\end{tabular}

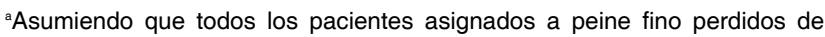
seguimiento no se curaron y que todos los pacientes asignados a pediculicidas perdidos del seguimiento sí se curaron.

\section{Conclusiones}

El uso de peine fino con instrucciones es más efectivo para combatir la pediculosis en la comunidad que el tratamiento con pediculicida.

Palabras clave: piojos de la cabeza, pediculus, permetrina, eficacia del tratamiento. Key words: head lice, pediculus, permetrine, treatment efficacy. Fuente de financiamiento y conflicto de intereses: Big Lottery Fund y The London School of Hygiene and Tropical Medicine.

\section{Comentario}

Tanto la prevalencia, como el grado de infestación por Pediculus humanus capitis (cuyo ciclo reproductivo es exclusivamente humano) está relacionado en forma inversa con el nivel socioeconómico . Por ejemplo, la prevalencia en niños escolarizados de la ciudad de La Rioja ${ }^{1}$ es del $61 \%$ y en comunidades indígenas de Misiones $^{2}$ es de $83 \%$, con una alta comorbilidad de eccema de cuello y cuero cabelludo. El $80 \%$ de los niños de escuelas públicas y privadas de Buenos Aires ha tenido pediculosis alguna vez, con una prevalencia mínima en verano $(12 \%)$ y máxima en invierno $(56,8 \%)$ y una media anual de $38,04 \%$ (IC95\%: $34-42,9)^{3}$. En los sectores medios y altos, los piojos se han hecho resistentes a los pediculicidas, mientras que en las poblaciones pobres, conservan mayor sensibilidad a estos productos. El aumento de la resistencia en este último tiempo a los productos pediculicidas del mercado (en especial los piretroides ${ }^{4}$, las drogas que cuentan con mayor evidencia científica de efectividad ${ }^{5}$ ) produjo un aumento de la infestación en los niños y ésta, a su vez, mayor uso de productos químicos con creciente concentración, lo que aumenta el riesgo de efectos adversos locales y/o acumulativos, como la neurotoxicidad. Con el objetivo de reducir la probabilidad de complicaciones como eccematización e impetiginización, se recomienda tratar al caso índice y a los convivientes con evidencia de infestación. Como recomienda el Centro de Control y Prevención de las Enfermedades de $\mathrm{EEUU}^{6}$ (en inglés $\mathrm{CDC}$ ) sólo deberían ser tratados con pediculicidas los individuos mayores de dos años en quienes se pueda documentar la presencia de piojos que se mueven, o de liendres a menos de $6 \mathrm{~mm}$ (el ancho del dedo meñique) del cuero cabelludo, lo que significa que todavía no han sido incubadas. Este estudio evalúa la eficacia de los tratamientos de la pediculosis en el contexto de condiciones realistas y de acuerdo a las instrucciones de los fabricantes.

\section{Conclusión de los comentadores}

Si bien no contamos con información sobre la costo-efectividad local de dicha estrategia, como todavía no existe un pediculicida ideal, el uso de peine fino parece una buena opción para combatir esta endemia ya que no genera resistencia, es barata y está exenta de efectos adversos.

Ariana Cheng y Sergio Terrasa [ Servicio de Medicina Familiar y Comunitaria del Hospital Italiano de Buenos Aires. ]

Recibido el 10/07/06 y aceptado el 20/12/06

Cheng A y Terrasa S. El peine fino sería más efectivo que los pediculicidas. Evid. actual. práct. ambul; 10(1):7, ene-feb.2007. Single blind, randomised, comparative study of the Bug Buster kit and over the counter pediculicide treatments against head lice in the United Kingdom. N Hill Head, G Moor, MM Cameron, A Butlin, S Preston, MS Williamson, C Bass. BMJ 2005; 331:384-387. PMID: 16085658. Disponible en URL: http://www.bmj.com/cgi/reprint/331/7513/384

\section{Referencias}

1. Catalá Silvia, Junco Luis, Vaporaky Rita. Pediculus capitis infestation according to sex and social factors in Argentina. Rev. Saúde Pública. [periódico en la Internet]. 2005 Jun [citado 2007 Ene 09]; 39(3): 438-443.

2. De Villalobos, C; Ranalletta, M; Rodríguez, A. Relevamiento de la pediculosis en dos comunidades indígenas de la provincia de Misiones. Arch. argent. dermatol; 47(4):191-6,1997.

3. Del Castro C., Abramovich A, Cicchino, A, et al. Prevalence and seasonal variation of Pediculosis capitis in the population under sixteen year of age of the health region of Buenos Aires, Argentina. Rev. Saúde Pública, ago. 1994, vol.28, no.4, p.295-299.

4. Picollo MI, Vassena CV, Mougabure Cueto G, Vernetti M, Zerba EN. Resistance to insecticides and effect of synergists on prmethrin toxicity in Pediculus capitis (Anoplura: Pediculidae) from Buenos Aires. J Med Entomol 2001; 37:721-5. Citado por1.

5. R Vander Stichele, E Dezeure, M Bogaert. Systematic review of clinical efficacy of topical treatments for head lice. BMJ 1995;311:604-608. Disponible en URL: http://www.bmj.com/cgi/content/full/311/7005/604 [último acceso: 09/01/2006].

6. US Centers for Disease Control and Prevention. Division of Parasitic Diseases. Fact sheet: treating head lice. Disponible en URL: www.cdc.gov/ncidod/dpd/parasites/headlice/factsht_head_lice_treating [último acceso: 09/01/2006]. 